\title{
Improved State of Charge Estimation for Lithium-Sulfur Batteries
}

\author{
Karsten Propp ${ }^{\mathrm{a}}$, Daniel J. Auger ${ }^{\mathrm{a}, *}$, Abbas Fotouhi $^{\mathrm{a}}$, Monica Marinescu ${ }^{\mathrm{b}}$, \\ Vaclav Knap ${ }^{c}$, Stefano Longo ${ }^{a}$ \\ ${ }^{a}$ School of Aerospace, Transport and Manufacturing, Cranfield University, Cranfield, Beds., MK43 OAL, UK \\ ${ }^{b}$ Department of Mechanical Engineering, Imperial College London, SW7 2AZ, UK \\ ${ }^{c}$ Department of Energy Technology, Aalborg University, Aalborg, 9000, Denmark
}

\begin{abstract}
Good state of charge estimation in lithium-sulfur batteries (Li-S) is vital, as the simplest convention methods commonly used in lithium-ion batteries - opencircuit voltage measurement and 'coulomb counting' - are often ineffective for Li-S. Since Li-S is a new battery chemistry, there are few published techniques. Existing techniques based on the extended Kalman filter and the unscented Kalman filter have shown some promise, existing work has explored only one of many possible estimator architectures: a single filter based on a precalibrated behavioural reparameterization of an equivalent circuit network whose parameters vary as a function of state of charge and temperature. Such filters have been shown to be reasonably effective in practical cases, but they can converge slowly if initial conditions are unknown, and they can become inaccurate with changes in current density. It is desirable to understand whether other possible estimator architectures offer improved performance. One such alternative architecture is the 'dual extended Kalman filter', which uses voltage and current measurements to estimate into a short-term dynamic circuit parameters then uses the outputs of this in a slower-acting state-ofcharge estimator. This paper develops a 'behavioural' form of the dual extended Kalman filter, and applies this to a lithium-sulfur battery. The estimator is
\end{abstract}

\footnotetext{
*Corresponding author

Email address: d. j.auger@cranf ield.ac.uk (Daniel J. Auger )
} 
adapted with a term to model circuit current dependence, and demonstrated using pulse-discharge tests and scaled automotive driving cycles including some with initially partially discharged batteries. Compared to the published state-of-the-art, the new estimators were are found to be between $16.4 \%$ and $28.2 \%$ more accurate for batteries that are initially partially discharged to a $60 \%$ SoC level; the new estimators also converge faster. The resulting estimators have the potential to be extended to state-of-health measures, and the 'behavioural' circuit reparameterization is likely to be of use for other battery chemistries beside lithium-sulfur.

Keywords: lithium-sulfur battery, state of charge estimation, extended Kalman filter, online parameterzation, equivalent circuit network model

\section{Introduction}

\subsection{Background and Context}

Lithium-sulfur (Li-S) batteries have the potential to offer lightweight, cheap and safe energy storage in the near future. There are problems that still need to be solved in order to realize Li-S's potential, such as relatively short cycle life, low charging efficiency and power capabilities [1, 2]. Another challenge for practical systems is the complex conversion reaction of elemental sulfur $\mathrm{S}_{8}$, via the intermediates $\mathrm{Li}_{2} \mathrm{~S}_{8}, \mathrm{Li}_{2} \mathrm{~S}_{4}, \mathrm{Li}_{2} \mathrm{~S}_{2}$, to lithium sulfide $\mathrm{Li}_{2} \mathrm{~S}$ [3] during discharge. Within the high plateau (see Fig. 11 top) soluble high order polysulfides $\left(\mathrm{L}_{2} \mathrm{~S}_{8}, \mathrm{Li}_{2} \mathrm{~S}_{6}\right)$ are predominant in electrolyte solution [4], which leads to a small internal resistance but also to self discharge due to the shuttle effect [5]. The low plateau is governed by insoluble species $\left(\mathrm{Li}_{2} \mathrm{~S}_{4}\right.$, $\left.\mathrm{Li}_{2} \mathrm{~S}_{3}\right)$ [6] that are likely to precipitate $\left(\mathrm{Li}_{2} \mathrm{~S}_{2}, \mathrm{Li}_{2} \mathrm{~S}\right)$ [7, 8]. Compared to Li-ion batteries, the reaction path of Li-S cells is more complex, leading to challenges for practical applications:

- Firstly, it is hard to predict the exact reaction path, as it depends on the current profile and the short term availability of charge per cycle 


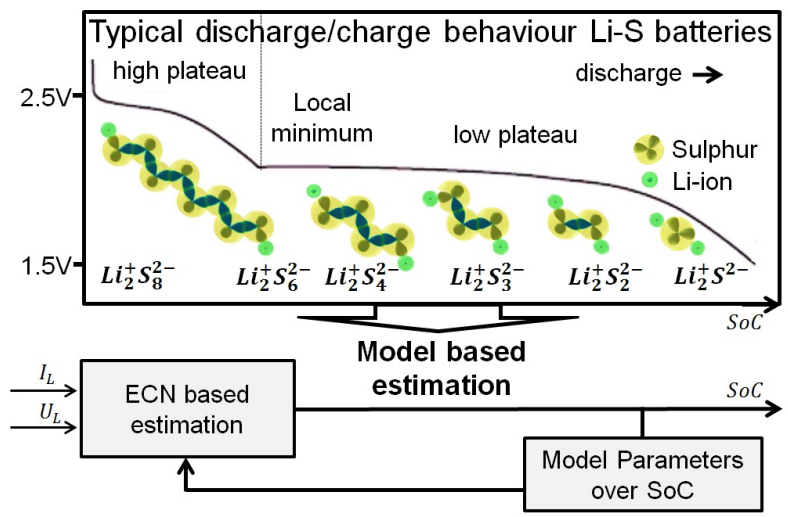

Alternative: Online Parameter estimation + SoC

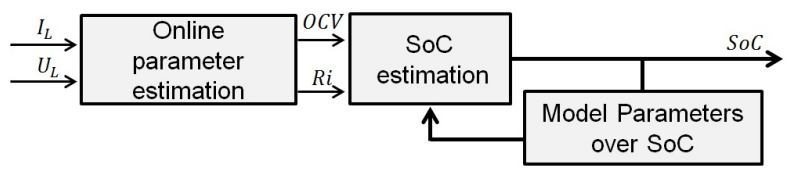

Figure 1: Behaviour and SoC estimation principles of Li-S batteries

can vary. The whole reaction path is by far less well understood than for Li-ion batteries [9]. Practically achieved capacities depend on the utilzation of the active sulfur within the cathode, which varies not only with current density, temperature and age, but also - significantly - on the current profile applied[10, 11, 12]. This can cause significant shortterm variations of the usable capacity, and in Li-S, and 'rated capacity' values determined at low constant discharge currents are rarely useful in practical applications.

- Secondly, the complex reaction path with many dissolved species existing in parallel makes it difficult to estimate the battery's state of charge. In some battery chemistries, this is easily determined from a monotonic relationship between state-of-charge and open-circuit voltage (OCV). In Li-S, the OCV curve has two voltage 'plateaus': a 'high' plateau, characterized by a low internal resistance, a monotonically decreasing OCV gradient and high rates of self discharge, and a 'low plateau' with a near-constant $\mathrm{OCV}$ and limited power capabilities appearing towards 
the end of discharge (Fig. 1). In contrast to many Li-ion batteries, in Li-S OCV is not an indicator for SoC over the whole discharge range. Furthermore, the power capability and degradation are difficult to predict accurately, since they are also less well understood and depend on cycling parameters [13]. While attempts have been made to model self discharge [14], capacity fade [15] and separate behaviour of both plateaus [16], to date, most of the complex chemical processes remain unmodelled for applications. That means that the validity of the currently existing models is compromised by ageing and cycling conditions [13].

- Thirdly, as Li-S batteries are a new technology, there few experiences with Li-S batteries in complex real world operating conditions and there is no widespread body of knowledge to be readily drawn upon.

At present, there is one published Kalman-filter technique for state estimation in Li-S batteries. This uses an 'behavioural' equivalent circuit network model dependent on temperature and state-of charge, derived from pulsedischarge tests [16]. This model is then incorporated directly into either an extended Kalman filter, an unscented Kalman filter or a particle filter. This is the first architecture illustrated in Fig. 1. and it is described in full in [17].

\subsection{Contribution and Relevance to Application}

As its key contribution, this paper presents an alternative estimator architecture for Li-S - the second illustrated in Fig. 1. In this, the problem of state of charge determination is broken into two:

- Online parameter estimation (from voltage and current).

- SoC estimation (from online parameter estimates).

It will be shown in this paper that this provides greater accuracy than the estimator described in [17]. The method published in this paper also contains a novel and effective technique for making the method robust to varying currents. 
This paper uses cycles representative of a target automotive application. This is order to ensure validity for practical applications: it is important that the current profiles applied to cells, modules and packs during testing are reasonably close to what would be seen in practice. (Simple constant discharge/charge profiles are unlikely to be a good representation of an electric passenger car.) Consideration is also given to practical implementation: the resulting methods are sufficiently computationally compact that they can be run as an embedded algorithm on a standard electronic control unit (ECU) such as might be incorporated in a battery management system (BMS).

\subsection{Outline}

This paper is structured in three main parts:

- Sec. 2 introduces the algorithm used for online parameter estimation. This uses an extended Kalman filter together with a behavioural reparameterization of a Thevenin model. This model is introduced briefly and employed for parameter identification. This is demonstrated on real Li-S data for two different automotive driving cycles.

- Sec. 3 explores the dependence of estimated parameters on current profiles with differing amplitudes; the relationship between current and estimated internal resistance is discussed in Sec. 4, and a reduced-order model of the dynamical resistance is developed in Sec. 5 .

- Sec. 6uses the results of the previous section to develop a SoC estimator, which is demonstrated for the two automotive driving cycles.

\section{Online parameter estimation via the extended Kalman filter}

A framework for online battery parameter estimation for operational conditions relevant to automotive applications (high current rates, temperature variations and dynamic rate profiles [18]) was proposed by Chiang et al. in 


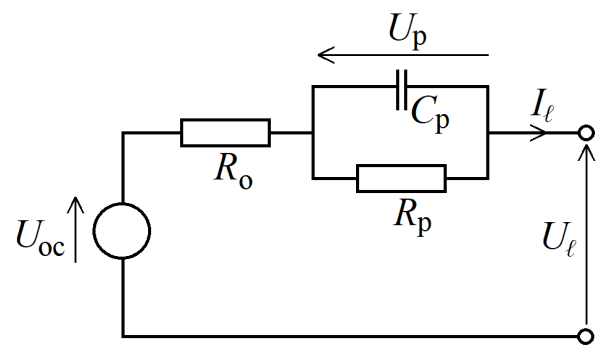

Figure 2: Thevenin model

[19]. There, an adaptive control method in combination with a Thevenin battery model [20], which has been proven to be a reasonable trade off between computational effort and accuracy [21, 22], is used to identify the OCV and internal resistance of lithium-iron-phosphate and lithium polymer cells. The mathematical assumptions made by Chiang were incorporated by He et al. to apply an adaptive Kalman filter-based online identification for realistic load profiles in electric vehicles [23, 24] . The literature for lithium-ion batteries uses a standard equivalent circuit network model, but in the literature the has been published on lithium-sulfur, good results for offline identification have been obtained using a behavioural reparameterization of that model [16]. A parameter estimator based on this behavioural reparameterization has not appeared in the literature to date, so it will be described here.

\subsection{Behavioural repararameterization of equivalent circuit network model}

The basis for the modelling in this work and many others is the well-known Thevenin model, illustrated in Figure 2. The Thevenin model describes the voltage at the battery terminal as

$$
U_{\ell}(t)=U_{\mathrm{oc}}-U_{\mathrm{p}}(t)-R_{\mathrm{o}} I_{\ell}(t)
$$

where the voltage over the $R C$ circuit is described as

$$
\dot{U}_{\mathrm{p}}(t)=-\frac{1}{R_{\mathrm{p}} C_{\mathrm{p}}} U_{\mathrm{p}}(t)+\frac{1}{C_{\mathrm{p}}} I_{\ell}(t)
$$


which are couched in terms of the components of the electrical equivalent circuit. As shown in [16], recasting the system in terms of the observed behaviours instead can help to separate the circuit parameters into an immediate part and a lagging part after a current pulse. Therefore, we will start by defining the corresponding parameters:

$$
\begin{aligned}
R_{\text {int }} & =R_{\mathrm{o}}+R_{\mathrm{p}}, \\
\rho=\frac{R_{\mathrm{p}}}{R_{\mathrm{o}}+R_{\mathrm{p}}} & \Leftrightarrow(1-\rho)=\frac{R_{\mathrm{o}}}{R_{\mathrm{o}}+R_{\mathrm{p}}}, \\
\Omega & =\frac{1}{R_{\mathrm{p}} C_{\mathrm{p}}} .
\end{aligned}
$$

$R_{\text {int }}$ is the steady-state (or quasi-static) internal resistance, $\rho$ is the fraction of the response that is not instantaneous, thus representing a dynamic lag and $\Omega$ is the natural frequency of the response. With this new set of parameters, equation (1) becomes

$$
U_{\ell}=U_{\mathrm{oc}}-U_{\mathrm{p}}-\underbrace{(1-\rho) R_{\mathrm{int}}}_{\text {formally } R_{\mathrm{o}}} I_{\ell}
$$

and equation (2) becomes

$$
\dot{U}_{p}=-\Omega U_{\mathrm{p}}+\rho R_{\text {int }} \Omega I_{\ell}
$$

Where the model parameters are $U_{\mathrm{oc}}$ for the OCV, $U_{\ell}$ for the terminal battery voltage and $U_{\mathrm{p}}$ for the voltage drop over the parallel $R C$ circuit. The main advantage of these transformation is to easily constrain the behavioural parameters to 'sensible' ranges since they are sufficiently decoupled from each other: the dynamic portion of the model can be adjusted without altering the steady-state response.

\subsection{Model formulation for the extended Kalman filter}

The behavioural model is particularly suitable for the online parameterization because it can simplify the parametrization of the covariance matrices of 
the EKF. Since the model contains more variables than observable states, there is no guarantee for the Kalman filter-based identification to converge towards the anticipated values [18]. In [23] the difficult parameterization of the EKF covariances is solved with an adaptive algorithm for estimating the covariance matrix for the system noise $Q$ and measurement noise $R$. Here, we assume that the statistics of measurement noise, and battery parameters are constant because it gives the user more flexibility to tune the filter. In the following, we adapt the assumptions made by Chiang et al. [19] for Li-S batteries and the behavioural model. We start with the standard Thevenin model's state transition equation for the terminal battery voltage $U_{L}$, the derivative of equation 1

$$
\dot{U}_{\ell}=\dot{U}_{\mathrm{oc}}-\dot{U}_{\mathrm{p}}-\dot{I}_{\ell} R_{\mathrm{o}}-I_{\ell} \dot{R}_{\mathrm{o}}
$$

Generally, the OCV of the battery is dependent on the SoC, the operating temperature $(T)$ and usage history $(h)$. Therefore, the corresponding definition of $U_{\mathrm{oc}}$ can be described as a function of SoC, $T, h$, whose derivative is

$$
\dot{U}_{\mathrm{oc}}=\frac{\delta U_{\mathrm{oc}}}{\delta S o C} \frac{\delta S o C}{\delta t}+\frac{\delta U_{\mathrm{oc}}}{\delta T} \frac{\delta T}{\delta t}+\frac{\delta U_{\mathrm{oc}}}{\delta h} \frac{\delta h}{\delta t} .
$$

For the representation of the battery in a discrete-time manner, time steps typically less than or equal to one second are often used. Due to the naturally slow rate of change of parameters of common Li-ion batteries, the equation is often simplified at each time step by a set of assumptions to remove parameter rates of change [19]; however, since Li-S batteries have different properties to common Li-ion batteries, , the applicability of these assumptions needs explicit consideration for the Li-S battery chemistry:

- $\frac{\delta S o C}{\delta t} \approx 0$ holds for a small change in battery charge

Li-S cells are operated under much lower $C$ rates, which makes this assumption more justified than in the case of Li-ion.

- $\frac{\delta U_{o c}}{\delta S o C} \approx 0$ in low plateau 
Furthermore, there is no change in OCV over the SoC within the low plateau, which approves the assumption.

- $\frac{\delta T}{\delta t} \approx 0$ when temperature changes slowly

The OCV of Li-S batteries depends more strongly on cell temperature [16] than the OCV of Li-ion. However, the rate of heat generation is assumed to be lower than that of heat dissipation, which is especially valid at low current rates and environmental control.

- $\frac{\delta h}{\delta t} \approx 0$ for long term history

Li-S cells are influenced by the short term discharge history [25, 26], commonly referred as the 'history' effect. The precise origins and extent for practical applications are still unexplored. However, it is expected that history effects occur at times longer than a time step of one second. Therefore $\dot{U}_{\mathrm{oc}}$ is approximated as 0 . Despite the relatively quickly changes of the internal resistance over the SoC between the two plateaus [16], we also assume it to be $0\left(\dot{R}_{0} \approx 0\right)$ for small periods. The conversion to the behavioural model can be done from the resulting change of the terminal voltage over time:

$$
\dot{U}_{\ell}=-\dot{U}_{\mathrm{p}}-R_{\mathrm{o}} \dot{I}_{\ell}
$$

Where the behavioural interpretation is

$$
\dot{U}_{\ell}=-\dot{U}_{\mathrm{p}}-(1-\rho) R_{\text {int }} \dot{I}_{\ell}
$$

for the terminal voltage and

$$
\dot{U}_{p}=-\Omega U_{\mathrm{p}}+\rho R_{\mathrm{int}} \Omega I_{\ell}
$$

for the voltage drop over the $R C$ circuit. By re-write equation 3 to bring $U_{p}$ on one side, substituting it into Eq. 9 and including the result for $\dot{U}_{\mathrm{p}}$ in Eq. 8 , the 
behavioural state transition equation can be derived as

$$
\dot{U}_{\ell}=\Omega U_{\mathrm{oc}}-\Omega U_{\ell}-\Omega R_{\mathrm{int}} I_{\ell}-(1-\rho) R_{\mathrm{int}} \dot{I}_{\ell} .
$$

\subsection{Implementation of the extended Kalman filter}

The Kalman filter is an efficient algorithm minimizing the error variance between the true and the estimated state. It is often applied for battery state estimation [27, 28] and for online parameter estimation [29]. The EKF is able to predict battery states, using a nonlinear system model $f$. In the following, the algorithm is summarized for a discrete system [30].

\section{Nonlinear state space model}

$x_{k}=f\left(x_{k-1}, u_{k-1}, w_{k-1}\right), \quad y_{k}=h\left(x_{k}, u_{k}, v_{k}\right)$.

\section{Definitions \\ $\hat{A}_{k}=\left.\frac{\partial f\left(x_{k}, u_{k}, w_{k}\right)}{\partial x_{k}}\right|_{x_{k}=\hat{x}_{k}^{+}}, \quad \hat{C}_{k}=\left.\frac{\partial h\left(x_{k}, u_{k}, v_{k}\right)}{\partial x_{k}}\right|_{x_{k}=\hat{x}_{k}^{-}}$.}

Initialisation for $k=0$

$\hat{x}_{0}^{+}=\mathbb{E}\left[x_{0}\right], \quad P_{0}^{+}=\mathbb{E}\left[\left(x_{0}-\hat{x}_{\mathrm{o}}^{+}\right)\left(x_{0}-\hat{x}_{0}^{+}\right)^{T}\right]$

\section{Computation EKF for $k=1,2, \ldots$}

State estimate update (assuming zero process noise): $\hat{x}_{k}^{-}=f\left(\hat{x}_{k-1}^{+}, u_{k-1}, 0\right)$

Error covariance update: $P_{k}^{-}=\hat{A}_{k-1} P_{k-1}^{+} \hat{A}_{k-1}^{T}+Q$

Output estimate (assuming zero measurement noise): $\hat{y}_{k}=h\left(\hat{x}_{k}^{-}, u_{k}, 0\right)$

Kalman Gain matrix: $L_{k}=P_{k}^{-} \hat{C}_{k}^{T}\left[\hat{C}_{k} P_{k}^{-} \hat{C}_{k}^{T}+R_{k}\right]^{-1}$

State estimate measurement update: $\hat{x}_{k}^{+}=\hat{x}_{k}^{-}+L_{k}\left[y_{k}-\hat{y}_{k}\right]$

Error covariance measurement update: $P_{k}^{+}=\left(I-L_{k} \hat{C}_{k}\right) P_{k}^{-}$

The state vector, describing the transition to the next time step for the parameters of the behavioural model is

$$
x=\left[\begin{array}{llllll}
U_{\mathrm{oc}} & U_{\ell} & U_{\mathrm{p}} & \Omega & \rho & R_{\mathrm{int}}
\end{array}\right]^{T} .
$$


The input $u$ is defined as the input current $\left(u:=I_{\ell}\right)$. The standard-form state equations can be populated from the equivalent-circuit network equations 9.10) with the assumption that the parameters vary only slowly with time: $\left(\dot{U}_{\mathrm{oc}} \approx 0, \dot{\Omega} \approx 0, \dot{\rho} \approx 0, \dot{R}_{\mathrm{int}} \approx 0\right)$.

The vector function describing the rate of change w.r.t. time of the state vector (11) assuming zero process noise is written:

$$
f(x, u)=\left[\begin{array}{llllll}
f_{U_{\mathrm{oc}}} & f_{U_{\ell}} & f_{U_{\mathrm{p}}} & f_{\Omega} & f_{\rho} & f_{R_{\mathrm{int}}}
\end{array}\right]^{T} .
$$

The individual functions model the nominal dynamics of each state. For the states that represent 'constant' (i.e. slowly varying) circuit parameters we have:

$$
\begin{array}{r}
f_{U_{\mathrm{oc}}}=\left.\frac{d U_{\mathrm{oc}}}{d t}\right|_{w=0}=0 \\
f_{\Omega}=\left.\frac{d \Omega}{d t}\right|_{w=0}=0 \\
f_{\rho}=\left.\frac{d \rho}{d t}\right|_{w=0}=0 \\
f_{R_{\mathrm{int}}}=\left.\frac{d R_{\mathrm{int}}}{d t}\right|_{w=0}=0
\end{array}
$$

The remaming equations come from the equivalent circuit network model equations, again assuming that since $w=0$, circuit parameters are sufficiently slowly variable that their derivatives can be treated as zero. From (10) we have:

$$
\begin{aligned}
f_{U_{\ell}} & =\left.\frac{d U_{\ell}}{d t}\right|_{w=0} \\
& \approx \Omega U_{\mathrm{oc}}-\Omega U_{\ell}-\Omega R_{\mathrm{int}} I_{\ell}-(1-\rho) R_{\mathrm{int}} \dot{I}_{\ell}
\end{aligned}
$$

From (9) we have:

$$
f_{U_{\mathrm{p}}}=\left.\frac{d U_{\ell}}{d t}\right|_{w=0} \approx-\Omega U_{\mathrm{p}}+\rho R_{\mathrm{int}} \Omega I_{\ell} .
$$

Since the measured terminal voltage of the battery is represented by the second 
state, the simplest measurement equation is

$$
h=U_{\ell}
$$

The system can be linearized about the current state vector by evaluating the Jacobians of $f(x, u)$ :

$$
\hat{F}=\left[\begin{array}{rrrccc}
0 & 0 & 0 & 0 & 0 & 0 \\
\Omega & -\Omega & 0 & \hat{F}_{2,4} & R_{\text {int }} \dot{I}_{\ell} & \hat{F}_{2,6} \\
0 & 0 & -\Omega & \hat{F}_{3,4} & R_{\text {int }} \Omega I_{\ell} & \rho \Omega I_{\ell} \\
0 & 0 & 0 & 0 & 0 & 0 \\
0 & 0 & 0 & 0 & 0 & 0 \\
0 & 0 & 0 & 0 & 0 & 0
\end{array}\right]
$$

where

$$
\begin{gathered}
\hat{F}_{2,4}=U_{\mathrm{oc}}-U_{\ell}-R_{\mathrm{int}} I_{\ell}, \\
\hat{F}_{2,6}=-\Omega I_{\ell}-(1-\rho) \dot{I}_{\ell}, \\
\hat{F}_{3,4}=-U_{\mathrm{p}}+\rho R_{\mathrm{int}} I_{\ell} .
\end{gathered}
$$

$\hat{A}$ is found by converting $F$ from continuous time to discrete time, e.g. by

$$
\hat{A} \approx I+T_{\mathrm{s}} \hat{F}
$$

where $T_{\mathrm{S}}$ is the sampling period. (Other well-known conversion methods, e.g. higher-order expansions, are equally permissible if computational resources allow.)

The measurement matrix $\hat{C}$ is obtained from:

$$
\begin{aligned}
\hat{C} & =\left[\begin{array}{llllll}
\frac{\partial h}{\partial U_{\mathrm{oc}}} & \frac{\partial h}{\partial U_{\ell}} & \frac{\partial h}{\partial U_{\mathrm{p}}} & \frac{\partial h}{\partial \Omega} & \frac{\partial h}{\partial \rho} & \frac{\partial h}{\partial R_{\text {int }}}
\end{array}\right] \\
& =\left[\begin{array}{llllll}
0 & 1 & 0 & 0 & 0 & 0
\end{array}\right]
\end{aligned}
$$


This model has a similar complexity to the models of section Sec.2 the tuning of the covariance matrices for process noise $w \sim(0, Q)$ and measurement noise $v_{k} \sim\left(0, R_{k}\right)$ is likely to be more intuitive. This will be elaborated on in section Sec. 2.4

\subsection{Experimental validation}

The validation of the proposed method is done by performing two experiments. Firstly, the Li-S battery model presented in [16] generates the voltage input for the EKF parameter estimation and the accuracy of the estimated parameters is evaluated by comparison to the known values from the model (Fig. 3 right). Secondly, the pulse test measurements and identification data from the offline parameterization (Fig. 3 left) are compared to the online method. In the following, the model parameters are shown in the 'conventional' ECN format, since as it is customary to map them back with

$$
R_{\mathrm{p}}=\rho_{\mathrm{p}} R_{\mathrm{int}}, \quad R_{\mathrm{o}}=R_{\mathrm{int}}-R_{\mathrm{p}}, \quad C_{\mathrm{p}}=\frac{1}{R_{\mathrm{p}} \Omega_{\mathrm{p}}} .
$$

The online parameterization uses the measurement error, i.e. the difference between the measured and predicted battery terminal voltage, to correct six parameters or states. While the model determines the separation into the parameters (OCV, $R_{\mathrm{o}}, R_{\mathrm{p}} C_{\mathrm{p}}$ ), the convergence to the right values also depend on the right choices for the system and measurement uncertainties. The values of $R$ and $Q$ determine the relative trust of model prediction and measurement, as these both can generally be ridden with errors. The presented values for $R=0.006, Q$ and $P_{0}$ were derived iteratively to follow the charge dependent changes without too many parameter fluctuations. They were found to deliver 
robust results for a wide range of experiments:

$$
\begin{gathered}
Q=\left[\begin{array}{cccccc}
0.02 & 0 & 0 & 0 & 0 & 0 \\
0 & 0.01 & 0 & 0 & 0 & 0 \\
0 & 0 & 0.01 & 0 & 0 & 0 \\
0 & 0 & 0 & 0.0002 & 0 & 0 \\
0 & 0 & 0 & 0 & 0.3 & 0 \\
0 & 0 & 0 & 0 & 0 & 0.005
\end{array}\right] \times 10^{-4} \\
P_{0}=\left[\begin{array}{cccccc}
0.02 & 0 & 0 & 0 & 0 & 0 \\
0 & 1 & 0 & 0 & 0 & 0 \\
0 & 0 & 1 & 0 & 0 & 0 \\
0 & 0 & 0 & 0.00001 & 0 & 0 \\
0 & 0 & 0 & 0 & 1 & 0 \\
0 & 0 & 0 & 0 & 0 & 1
\end{array}\right]
\end{gathered}
$$

The initial values for the state vector are chosen to be close but not identical to the known values of a fully charged Li-S battery, to account for a level of uncertainty between different batteries.

$$
x_{0}=\left[\begin{array}{llllll}
2.43 \mathrm{~V} & 2.43 \mathrm{~V} & 0 \mathrm{~V} & 0.025 & 0.1 & 0.172 \Omega
\end{array}\right]^{T}
$$

\subsubsection{Parameter identification results}

For the simulation of the terminal voltage with the known data from the battery model, the realistic but simple NEDC current profile [31, 32] is used (Fig. 3 left). The identification is able to quickly adjust to the right values, even if the initial conditions for $R_{0}, R_{\mathrm{p}}$ and $C_{\mathrm{p}}$ are incorrect. Especially the OCV and $R_{0}$ can be identified precisely and without fluctuations, which makes this algorithm suitable for the SoC estimation (Sec.6). 


\subsubsection{Comparison with offline parameterization methods}

The offline identification data of the cell parameters over SoC was gathered measuring the response of 3.4 Ah OXIS Energy long life chemistry cells to a mixed current pulse profile (300 mA, $1450 \mathrm{~mA}$ and $2900 \mathrm{~mA})$ [16]. In order to identify current-related changes, the identification was done for each pulse individually with a window of $300 \mathrm{~s}$ before and after. For parameter identification, the prediction error minimization (PEM) algorithm, which is described in full detail by Ljung [33].
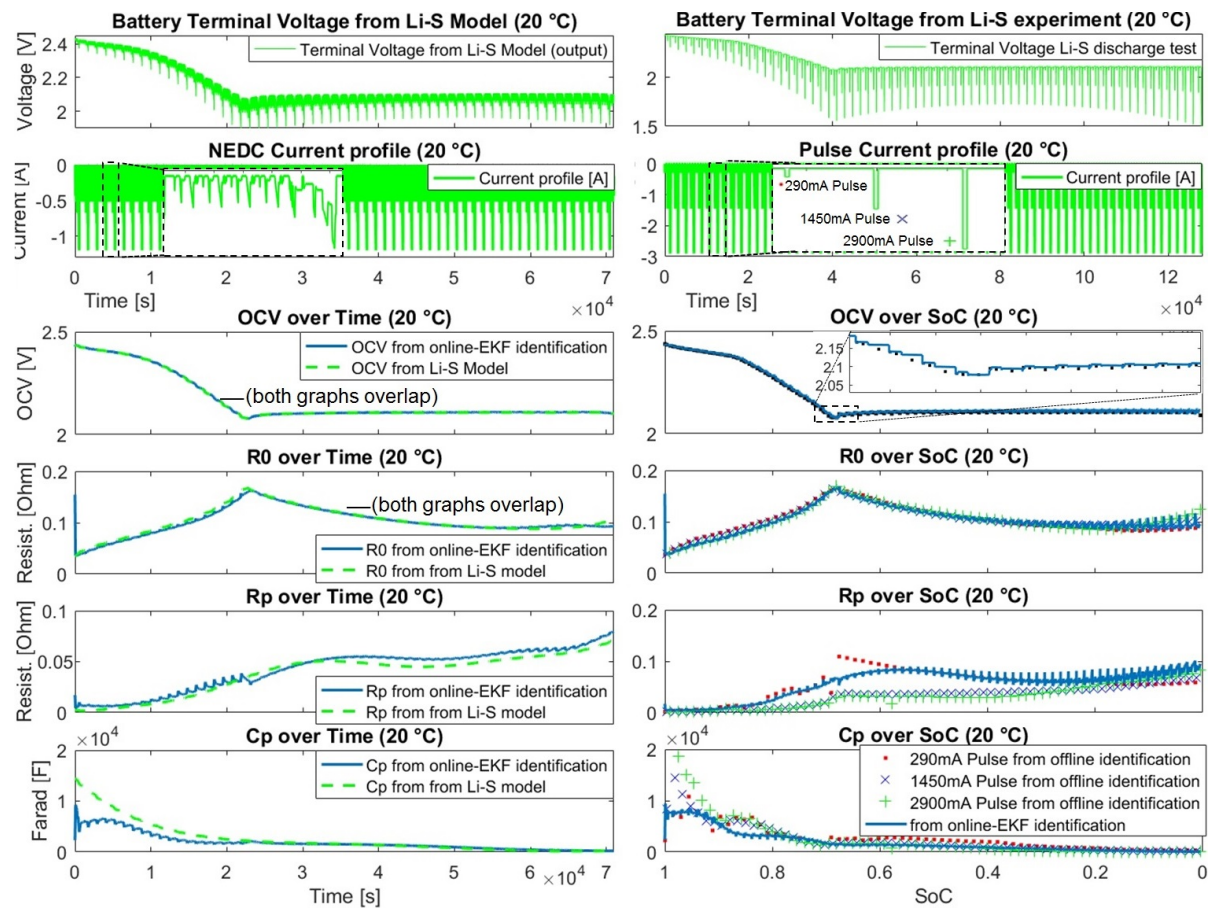

Figure 3: Online parameter identification results

The parametrization results, presented in Fig. 3 right, illustrate that, despite a significant improvement in computational time, the online estimation with the EKF provides a comparable identification quality to the offline methods. The zoomed in area demonstrates that parameter variations can be followed accurately, expecially for the OCV and $R_{\mathrm{O}}$. The EKF-based estimation method 
appears valid for the whole discharge range of Li-S batteries.

\section{Application of online parameter estimator to driving cycle scenarios}

The new EKF online parameterization method has been applied to two realistic scenarios. Current profiles representing the New European Drive Cycle (NEDC) [31] and the Urban Dynamometer Driving Schedule (UDDS) [34] were used in a controlled environment at $20{ }^{\circ} \mathrm{C}$ (Thermal Chamber: Memmert ICP260). Two cells were individually tested to reduce the impact of manufacturing variations. The tested cells are pre-cycled $(C / 10$ charge, $C / 5$ discharge, $\left.30^{\circ} \mathrm{C}\right)$ 3.4 Ah Li-S long life chemistry cells, developed by OXIS Energy.

To represent variation in power demands, each of the two current profiles (NEDC and UDDS) has been scaled with three different gains. This allows the testing of both the batteries behaviour as well as the SoC estimation performance with different power demands (Fig. 4). Since both cells were found to exhibit the same behaviours, for clarity, we have presented the results of cell 1 in Sec. 4 , Sec. 5 and most of Sec. 6 . However, tests from cell 2 in Sec. 6.4 The details of the six tests and the measured usable capacities of both cells are summarized in Table 1

Table 1: Discharge experiments

\begin{tabular}{lccc}
\hline Cycle & Cap. Cell 1 & Cap. Cell 2 & Av. Current \\
\hline NEDC 1.2 & $2.93 \mathrm{Ah}$ & $2.98 \mathrm{Ah}$ & $0.15 \mathrm{~A}$ \\
NEDC 1.8 & $2.69 \mathrm{Ah}$ & $2.68 \mathrm{Ah}$ & $0.22 \mathrm{~A}$ \\
NEDC 2.2 & $2.52 \mathrm{Ah}$ & $2.63 \mathrm{Ah}$ & $0.30 \mathrm{~A}$ \\
UDDS 1.2 & 3.11 Ah & $3.13 \mathrm{Ah}$ & $0.13 \mathrm{~A}$ \\
UDDS 1.8 & $2.88 \mathrm{Ah}$ & $3.07 \mathrm{Ah}$ & $0.19 \mathrm{~A}$ \\
UDDS 2.2 & 2.58 Ah & $2.53 \mathrm{Ah}$ & $0.26 \mathrm{~A}$ \\
\hline
\end{tabular}

For all the tests the capacities and reference SoCs are calculated by Coulomb counting for each test separately. This is done by integrating the measured current, following the cell's recommended voltage range between $2.45 \mathrm{~V}$ (SoC 
$=100 \%)$ and $1.5 \mathrm{~V}(\mathrm{SoC}=0 \%)$

$$
\text { SoC }=\left.S o C\right|_{t=0}-\frac{1}{3600 Q_{\text {cap }}} \int_{0}^{t} i(\tau) d \tau \text {. }
$$

Generally it is difficult to predict the capacity of the cell with Coulomb counting. However, it can be calculated retrospectively for a given voltage window and used as reference SoC in theory. This means that factors like self discharge and capacity changes are included within the reference SoC, which makes it a precise practical value for each scenario respectively. 

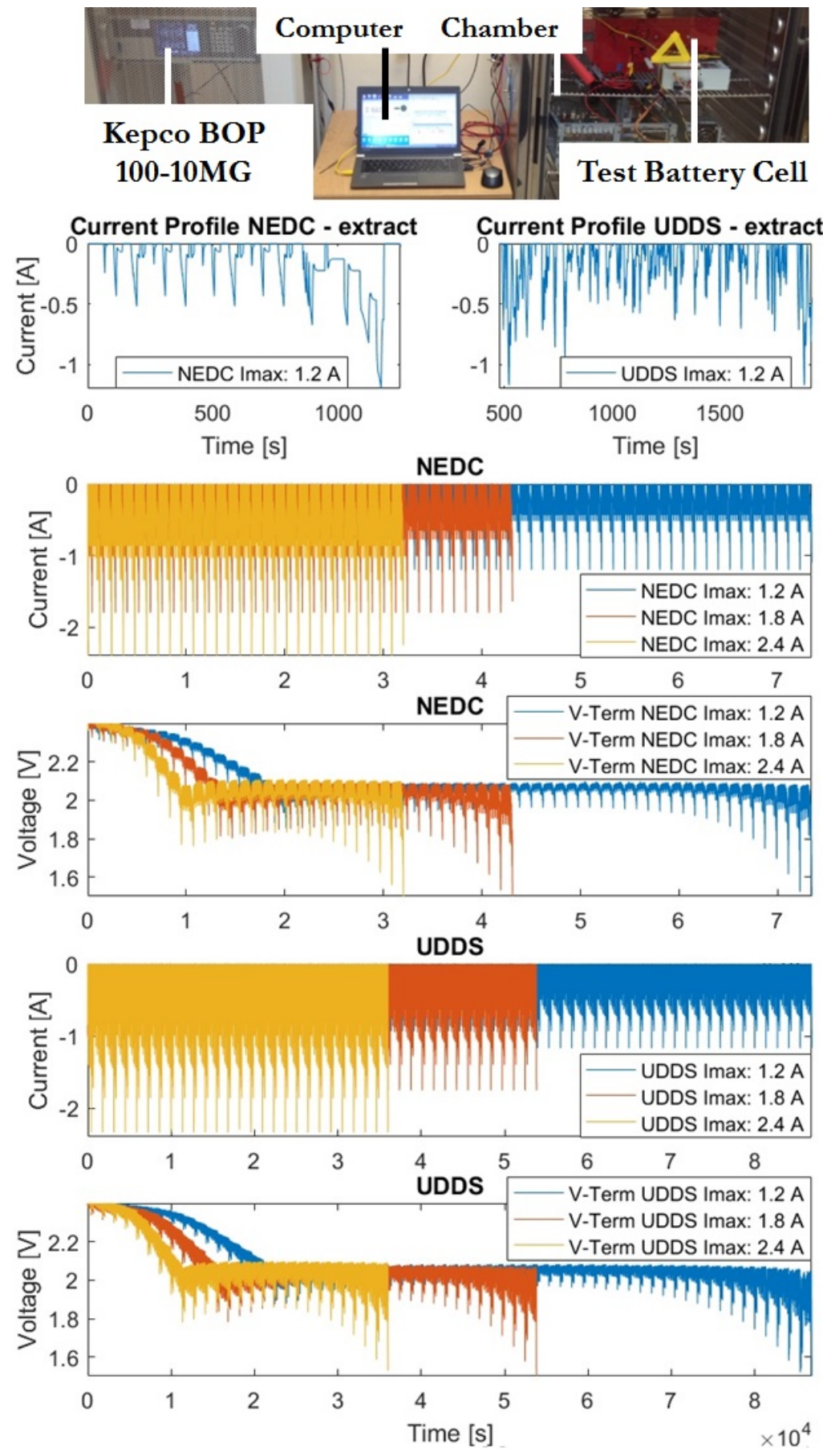

Figure 4: Experimental set-up and applied discharge currents 


\section{Relationship between current and online estimates of internal resistance}

The results of the parameter estimation over time for all current profiles are shown in Fig. 5
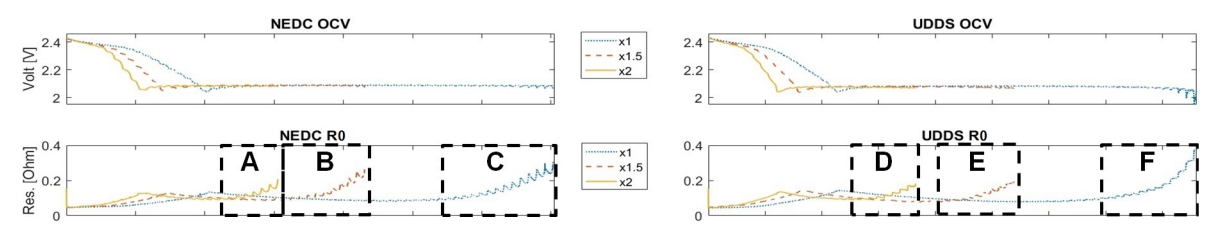

NEDC R1

UDDS R1
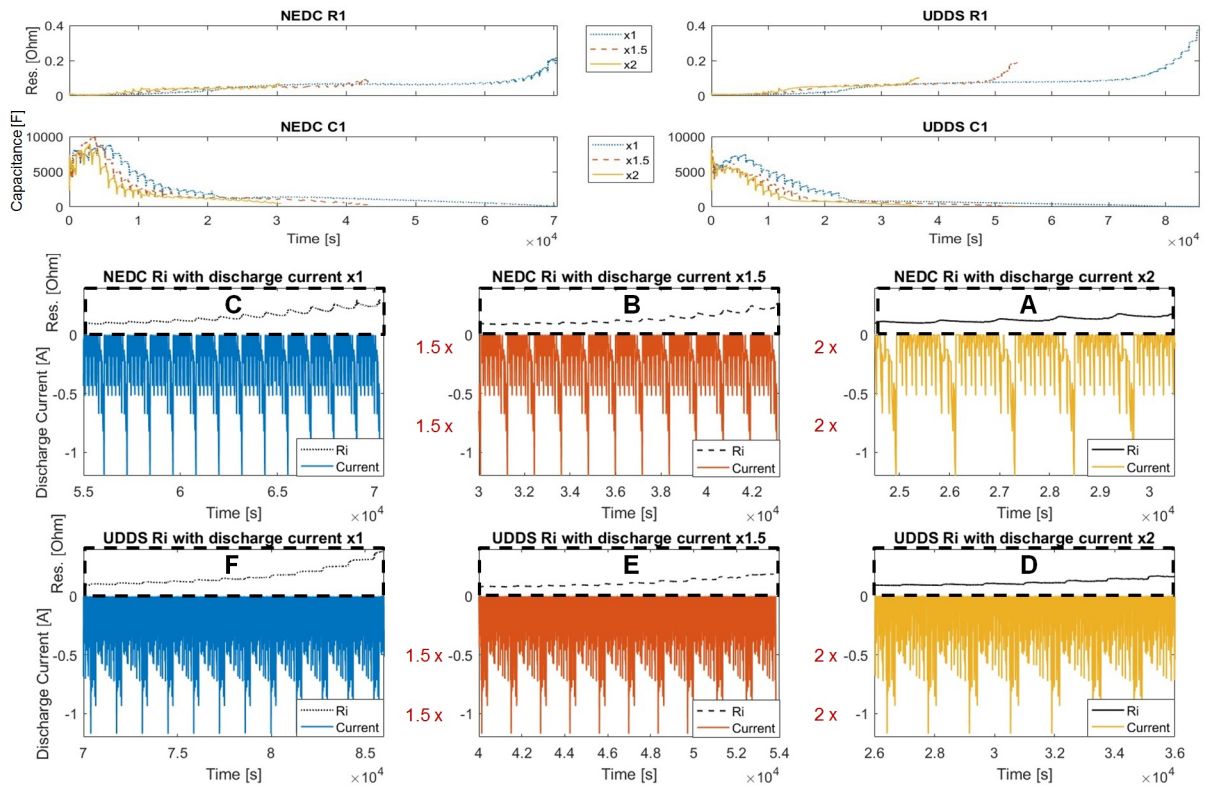

Figure 5: EKF online parametrization results for different current profiles

Generally, the values of the online identification correspond well to the offline obtained values presented in Fig. 3. However, the internal resistance of the cell, $R_{\mathrm{O}}$, has a different pattern. Towards the end of discharge $R_{\mathrm{O}}$ rises more quickly than predicted by the pulse discharge tests [16] and shows a relationship with the applied current density. The enlarged areas in Fig. 5 show the normalized discharge current together with the identified $R_{\mathrm{o}}$. While higher rates increase the internal resistance, lower currents or relaxation periods lead to a decrease in resistance. This is particularly visible in the NEDC cycle test. The more uniform currents in the UDDS cycle, containing less relaxation 
periods, unveil a more persistent growth of $R_{\mathrm{o}}$. Furthermore, it is visible that while the internal resistance rises more quickly with higher currents, larger values are reached with smaller rates towards the end of discharge. Here, the high currents cause the battery voltage to drop to the cut-of voltage of $1.5 \mathrm{~V}$ before the resistance values can grow further. The current dependencies in the observed $R_{0}$ suggest that it contains, next to the resistance of the electrolyte and current collectors [35], a diffusion part, which has been also reported and modelled in [36, 37] to fit impedance spectroscopy data. Here, high currents build up stronger gradients of active species in the electrolyte and fewer of them are available at the cathode surface to undergo the reaction. The building up of internal gradients could also explain why this effect has not been found as pronounced as here before. Common techniques, using identification windows [38] with artificial current pulses [39, 16], leave long resting periods in between the pulses to allow the battery to return to equilibrium state. The relatively short current pulses, applied after this resting period, are therefore not enough to build up the concentration gradients, observed in this study. Because the pulses are not able to sufficiently represent a realistic case for most applications, the data based on the continuously running EKF estimation method in combination with realistic current profiles is used to enhance the existing Li-S cell model.

\section{Modelling the dynamic internal resistance for SoC estimation}

The existing methods for parameter based SoC estimation for classic Li-ion batteries use the relationship between (online identified) OCV and SoC [19], since it contains sufficient information [40]. This is not the case for Li-S batteries [41], which makes it necessary to employ another source of information. We propose the combined information of OCV and internal resistance in a dual Kalman filter, one estimating the OCV and $R_{\mathrm{O}}$ and the other using them for the SoC estimation. For this we need a model predicting the internal resistance over the discharge range as seen in previous section. A useful bit of information 
from the online identification is where the existing Thevenin model for the pulse discharges is sufficient and where insufficient. For higher SoCs the fluctuations of internal resistance are small, indicating a reasonable model structure for the observations. The differences and fluctuations are mostly located in the last third of the discharge. Therefore, we use the existing model and suggest a simple addition of a dynamic resistance term $R_{\text {diff }}$. To fit the SoC estimation model to the observations, the previously used $R_{\mathrm{o}}$ is separated into a charge transfer $R_{\mathrm{ct}}$ and a diffusion $R_{\text {diff }}$ part. While $R_{\mathrm{ct}}$ is similar to the existing model, $R_{\text {diff }}$ is parametrized to reproduce the fluctuations of the internal resistance towards the end of discharge. To reproduce the observed behaviour of the internal resistance, we add a first order differential equation with current as input and a dynamic resistance as output. Mathematically this is similar to adding another parallel $R C$ pair to the model, which is not necessarily the most straight forward way to model the diffusion resistance. However, here we remind the reader that our focus is to reproduce the dynamic behaviour of the online identification results for the SoC estimation and not a physically detailed model. Therefore, we implement the current dynamics through a simple additional state within the existing battery model [16]. The internal resistance is represented by

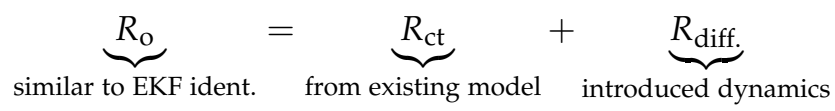

Where $R_{\text {diff }}$ is represented by

$$
\dot{R}_{\text {diff }}=\frac{1}{R_{\mathrm{D}} C_{\mathrm{D}}} R_{\text {diff }}+\frac{1}{C_{\mathrm{D}}} I_{\ell}
$$

$R_{\mathrm{D}}$ and $C_{\mathrm{D}}$ are varying parameters over SoC, with no physical meaning. They are chosen to represent the dynamic changes of the internal resistance over time in a similar manner to the identified values. For their parametrization we subtract the static $R_{\mathrm{O}}$, known from the model in [16] for $20^{\circ} \mathrm{C}$ of the internal 
resistance identified by the EKF.

$$
R_{\text {diff }}=R_{\mathrm{o}}-R_{\mathrm{ct}}
$$

The result (Fig. 6 A) shows the differences between the model parametrized by pulse data and the online EKF method. Since the main purpose of the identification is to capture the increase in $R_{\text {diff }}$ towards the end of discharge, the parametrization of the dimensionless factors $R_{\mathrm{D}}$ and $C_{\mathrm{D}}$ is only done for the positive values. The methodology is similar to the parameter identification is Sec. 2. but simplified. Again we chose a behavioural interpretation of Eq. 29 and change the parameters to a steady state value and a time constant

$$
\dot{R}_{\text {diff }}=-\Omega_{\mathrm{R}} R_{\text {diff }}+\Omega_{\mathrm{R}} R_{\mathrm{D}} I_{\ell}
$$

where $\Omega_{R}$ is

$$
\Omega_{\mathrm{R}}=\frac{1}{R_{\mathrm{D}} C_{\mathrm{D}}}
$$

The state vector becomes,

$$
x=\left[\begin{array}{lll}
R_{\text {diff }} & \Omega_{\mathrm{R}} & R_{\mathrm{D}}
\end{array}\right]^{T}
$$

and the state transition functions

$$
f(x, u)=\left[\begin{array}{lll}
f_{1} & f_{2} & f_{3}
\end{array}\right]^{T} .
$$

Where $f_{1}$ to $f_{3}$ are

$$
f_{1}=-x_{2} x_{1}+x_{2} x_{3} u, \quad f_{2}=0, \quad f_{3}=0,
$$

similar to the $R_{\mathrm{p}}$ and $C_{\mathrm{p}}$ values defined in section Sec. 2. The measurement equation is

$$
h=x_{1}
$$


The Jacobians are:

$$
\begin{gathered}
\hat{A}=\left[\begin{array}{ccc}
-x_{2} & -x_{1}+x_{3} u & x_{2} u \\
0 & 0 & 0 \\
0 & 0 & 0
\end{array}\right] \\
\hat{C}=\left[\begin{array}{lll}
1 & 0 & 0
\end{array}\right] .
\end{gathered}
$$

To simplify the presented model, all curves from the drive cycles and current densities are combined to one function over SoC (Matlab fitting tool [42]) for $R_{\mathrm{D}}$ and $C_{\mathrm{D}}$ respectively to

$$
f_{R_{\mathrm{D}}}(S o C)=0.9148 e^{(-10.79 S o C)}
$$

and

$$
f_{C_{\mathrm{D}}}(\text { SoC })=3071 e^{(5.03650 C)}
$$

Therefore, the dynamic part has nearly no effect for high SoCs, but becomes increasingly visible towards the end of discharge. The results of the improved model for the internal resistance are presented in Fig. 6 B, together with the parameter identification results. The dynamic part $R_{\text {diff }}$ and the static part $R_{\mathrm{O}}$ are plotted separately to show the effects of different currents for each part individually. Generally the added dynamic internal resistance supplements the model well. However, for the lowest applied current density the model cannot represent the growth of internal resistance as well as for the other scenarios. This is due to the simplifications made and to some factors playing a role at relatively low rates, such as precipitation [43]. For the SoC estimation we accept this error in order to maintain the model's simplicity. 

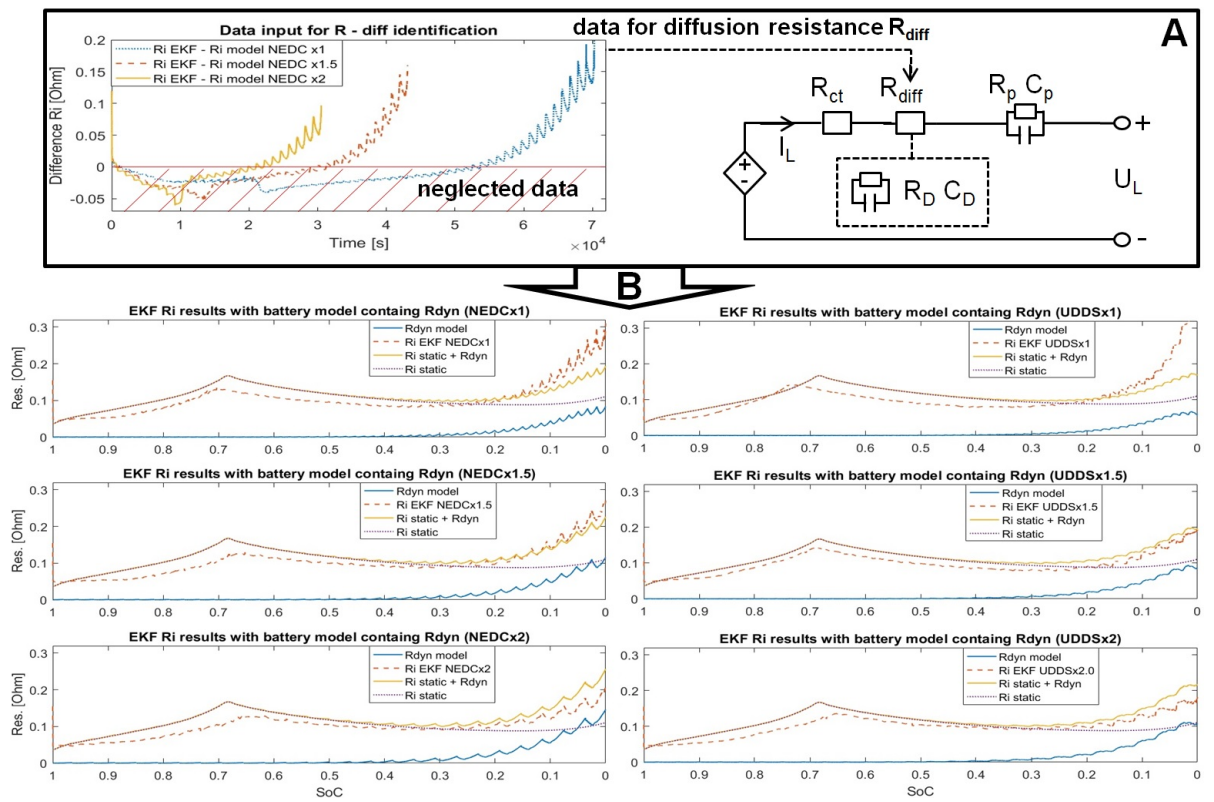

Figure 6: Modelling of $R_{\text {diff: }}$ Parametrizing $R_{\mathrm{D}}$ and $C_{\mathrm{D}}(\mathrm{A})$, Comparison of identification results and dynamic resistance model $R_{\mathrm{O}}+R_{\text {diff }}$ (B)

\section{Using online parameter estimates for state of charge estimation}

Now that we derived estimates of two necessary parameters, the OCV and $R_{\mathrm{O}}$, and a reasonable assumption about their behaviour over the discharge range for practical applications, we can derive the second Kalman filter. The main purpose of this EKF is to estimate a reasonable SoC from the batty model parameters. The battery model presented in [16] uses two polynomial functions for the static resistance $R_{\mathrm{ct}}$ and OCV respectively to represent the battery behaviour over the discharge range. For the sake of completeness they are presented as follows

$$
\begin{array}{r}
f_{\mathrm{OCV}}(S o C)=\left(1-\gamma_{\mathrm{m}, \mathrm{c}}(S o C)\right) f_{\mathrm{OCV}-\mathrm{low}}(S o C) \\
+\gamma_{\mathrm{m}, \mathrm{c}}(S o C) f_{\mathrm{OCV}-\mathrm{high}}(S o C)
\end{array}
$$


and $R_{\mathrm{ct}}$

$$
\begin{aligned}
f_{\text {Rct }}(S o C)=(1 & \left.-\gamma_{\mathrm{m}, \mathrm{c}}(S o C)\right) f_{\text {Rct-low }}(S o C) \\
& +\gamma_{\mathrm{m}, \mathrm{c}}(S o C) f_{\text {Rct-high }}(S o C) .
\end{aligned}
$$

The two polynomial functions are combined smoothly via a partial sinusoidal differentiable function $\gamma$ :

$$
\gamma_{\mathrm{m}, \mathrm{c}}(S o C):=\left\{\begin{array}{cl}
0, & \text { if a } \\
\frac{1}{2}+\frac{1}{2} \sin (2 m(S o C-c)) & \text { if } \mathrm{b} \\
1 & \text { if } \mathrm{c},
\end{array}\right.
$$

where the conditions $a, b, c$ stand for the different ranges,

$$
\begin{aligned}
\mathrm{a}: 2 m(\mathrm{SoC}-c)<-\frac{1}{2} \pi, \\
\mathrm{b}:-\frac{1}{2} \pi \leq 2 m(\mathrm{SoC}-c)<\frac{1}{2} \pi, \\
\mathrm{c}: 2 m(\mathrm{SoC}-c)>\frac{1}{2} \pi .
\end{aligned}
$$

Here $m$ is a scaling factor for the maximal gradient of the sinusoidal function, determining the transition region between the polynomials and $c$ represents the point where both functions are equally represented. Once the static internal resistance and OCV are defined over the discharge range, the dynamic internal resistance $R_{\text {diff }}$ can be included as a state for the SoC estimating EKF. This leads to the following state space model

$$
\begin{gathered}
\dot{x}(t)=A(t) x(t)+B(t) u(t) \\
y(t)=C(t) x(t)+D(t) u(t) .
\end{gathered}
$$

The dynamic states $x=\left[\begin{array}{ll}x_{1} & x_{2}\end{array}\right]^{T}$ of the system are the dynamic internal resistance $R_{\text {diff, }}$, as presented in previous section, and the SoC, calculated through Coulomb counting. The corresponding state space representation 
gives

$$
\begin{gathered}
A=\left[\begin{array}{cc}
\frac{-1}{f_{R_{\mathrm{D}}}\left(x_{2}\right) f_{\mathcal{C}_{\mathrm{D}}\left(x_{2}\right)}} & 0 \\
0 & 0
\end{array}\right] \quad B=\left[\begin{array}{c}
\frac{1}{f_{\mathrm{C}_{\mathrm{D}}}\left(x_{2}\right)} \\
\frac{-1}{3600 Q_{\mathrm{cap}}}
\end{array}\right] \\
C=\left[\begin{array}{cc}
1 & f_{R_{\mathrm{ct}}}\left(x_{2}\right) \\
0 & f_{O C V}\left(x_{2}\right)
\end{array}\right]
\end{gathered}
$$

with the current $I_{\ell}$ as an input.

\subsection{State of charge estimation with dual EKF}

For the application of the EKF algorithm with the presented Li-S battery model, the Jacobians of the matrices A and C are needed. With the relating functions over SoC, denoted as $x_{2}$, we therefore need the derivatives of $f_{\mathrm{OCV}}\left(x_{2}\right), f_{R_{\mathrm{ct}}}\left(x_{2}\right), f_{R_{D}}\left(x_{2}\right)$ and $f_{C_{D}}\left(x_{2}\right)$. Using one exponential function for each $R_{\mathrm{D}}$ and $C_{\mathrm{D}}$ the Jacobian matrix of $\mathrm{A}$ is obtained as follows:

$$
\begin{gathered}
\hat{A}(1,1)=\frac{-1}{f_{C_{\mathrm{D}}}\left(x_{2}\right) f_{R_{\mathrm{D}}}\left(x_{2}\right)} \quad \hat{A}(2,1)=0 \quad \hat{A}(2,2)=0 \\
\hat{A}(1,2)=\left[\frac{\dot{f}_{C_{\mathrm{D}}}\left(x_{2}\right)}{f_{C_{\mathrm{D}}}\left(x_{2}\right)^{2} f_{R_{\mathrm{D}}}\left(x_{2}\right)}\right. \\
\left.+\frac{\dot{f}_{R_{\mathrm{D}}}\left(x_{2}\right)}{f_{C_{\mathrm{D}}}\left(x_{2}\right) f_{R_{\mathrm{D}}}\left(x_{2}\right)^{2}}\right] x_{1} \\
-\left[\frac{\dot{f}_{C_{\mathrm{D}}}\left(x_{2}\right)}{f_{C_{\mathrm{D}}}\left(x_{2}\right)^{2}}\right] I_{\text {Bat }}
\end{gathered}
$$

where $\dot{f}_{R_{\mathrm{D}}}\left(x_{2}\right)$ and $\dot{f}_{C_{\mathrm{D}}}\left(x_{2}\right)$ are simply the first derivations of Eq. 39 and Eq. 40, defined as

$$
\begin{gathered}
\dot{f}_{R_{\mathrm{D}}}\left(x_{2}\right)=-9.875 e^{\left(-10.79 x_{2}\right)} \\
\dot{f}_{C_{\mathrm{D}}}\left(x_{2}\right)=15465.556 e^{\left(5.036 x_{2}\right)}
\end{gathered}
$$


Following the same principle for $C$, its Jacobian matrix is obtained as

$$
\begin{gathered}
\hat{C}(1,1)=1 \quad \hat{C}(1,2)=\dot{f}_{R_{c t}}\left(x_{2}\right) \\
\hat{C}(2,1)=0 \quad \hat{C}(2,2)=\dot{f}_{\text {OCV }}\left(x_{2}\right) .
\end{gathered}
$$

The derivatives of the combined functions $\dot{f}_{\mathrm{OCV}}$ and $\dot{f}_{\mathrm{R}_{\mathrm{ct}}}$ with respect to $x_{2}$ are influenced by the introduced factor $\gamma$, here substituted by $f_{\gamma}\left(x_{2}\right)$.

$$
\begin{array}{r}
f_{\mathrm{OCV}}\left(x_{2}\right)=\left(1-f_{\gamma}\left(x_{2}\right)\right) f_{\mathrm{OCV}-\mathrm{low}}\left(x_{2}\right) \\
+f_{\gamma}\left(x_{2}\right) f_{\mathrm{OCV}-\operatorname{high}}\left(x_{2}\right)
\end{array}
$$

Derivation with respect to $x_{2}$ leads to

$$
\begin{aligned}
\dot{f}_{\mathrm{OCV}}\left(x_{2}\right)=\dot{f}_{\mathrm{OCV}-\mathrm{low}}\left(x_{2}\right) & -\left(\dot{f}_{\gamma}\left(x_{2}\right) f_{\mathrm{OCV}-\mathrm{low}}\left(x_{2}\right)\right. \\
& \left.+f_{\gamma}\left(x_{2}\right) \dot{f}_{\mathrm{OCV}-\mathrm{low}}\left(x_{2}\right)\right) \\
& +\dot{f}_{\gamma}\left(x_{2}\right) f_{\mathrm{OCV}-\mathrm{high}}\left(x_{2}\right) \\
& +f_{\gamma}\left(x_{2}\right) \dot{f}_{\mathrm{OCV}-\mathrm{high}}\left(x_{2}\right) .
\end{aligned}
$$

Where $\dot{f}_{\gamma}$ is defined by

$$
\dot{\gamma}_{m, c}\left(x_{2}\right):=\left\{\begin{array}{cc}
0, & \text { if } \mathrm{a} \\
m \cos \left(2 m\left(x_{2}-c\right)\right), & \text { if } \mathrm{b} \\
0, & \text { if } \mathrm{c}
\end{array}\right.
$$

with same conditions for $a, b, c$ as in Eq. 44. The derivation of the static internal resistance $R_{c t}$, given by the Eq. 42 , follows the same pattern and is not presented. Instead, the derivation of the covariance matrices $R$ and $Q$ is examined thoroughly. In the process of finding the covariances, values were found that improve the convergence time for wrong initial conditions within the high plateau and values were found enhancing the correct estimation towards the stable low plateau of the discharge range. As an advantage, the dual filter offers the opportunity to distinguish between the high and low 
plateau, simply by evaluating the identified OCV. Therefore, $R$ and $Q$ are varied between the plateaus, using an 'if' function included in the second EKF. If the identified OCV is larger than $2.15 \mathrm{~V}, Q$ and $R$ are emphasized on the OCV identification and if the identified OCV is lower than $2.15 \mathrm{~V}, Q$ and $R$ rely on the results for Coulomb counting and the internal resistance. The resulting the parameterization of the covariance matrices for the two main discharge regions were derived iteratively. They are chosen towards a quick convergence in the high plateau and a reasonable stable result within the lower plateau. The values are

$$
R_{\text {high }}=\left[\begin{array}{cc}
0.15 & 0 \\
0 & 0.00054
\end{array}\right], Q_{\text {high }}=\left[\begin{array}{cc}
0.1 & 0 \\
0 & 0.01
\end{array}\right]
$$

for the high plateau and

$$
R_{\text {low }}=\left[\begin{array}{cc}
0.00015 & 0 \\
0 & 0.549
\end{array}\right], Q_{\text {low }}=\left[\begin{array}{cc}
0.1 & 0 \\
0 & 0.0000001
\end{array}\right]
$$

within the low plateau. In both cases the initial condition for the probabilities $P$ is

$$
P_{0}=\left[\begin{array}{cc}
10 & 0 \\
0 & 10
\end{array}\right]
$$

\subsection{SoC estimation with an initially fully charged battery}

The results of the proposed SoC estimation algorithms are evaluated by their convergence time, tested with imprecise initial values for the SoC state, and their estimation accuracy, measured by the root mean squared error (RMSE) over the whole discharge range

$$
\text { RMSE }=\frac{1}{\sqrt{n}}\left(\sum_{i=1}^{n}\left(S o C_{t, i}-S \hat{o} C_{t, i}\right)^{2}\right)^{0.5} .
$$

Where $n$ is the number of data points, $S o C_{t, i}$ is the reference SoC from the measurement and $S \hat{o} C_{t, i}$ is the estimated $\mathrm{SoC}$ by the proposed technique. 
Since the model does not include a current rate dependency for the discharge capacity of Li-S cells, the presented SoC estimator uses the identified capacity of 9778 As (2.72 Ah) for all applied currents. This value has been obtained from a pulse current test at $20^{\circ} \mathrm{C}$ in [16] and used for SoC estimation in [17]. Therefore, it allows the comparability to previously suggested SoC estimation methods. The initial conditions for the parameter identification part of the estimation are similar for every example and the same as in Eq. 26.

The achieved actual cell capacities in the drive cycle tests vary from 2.52 Ah (for NEDC) to 3.11 Ah (for UDDS), which is a variation of $21 \%$ compared to the rated capacity of the cell model. These variations between actual cell capacity and predicted one is another indicator for the insufficiency of Coulomb counting on its own for SoC estimation with the tested cells.

However, the estimation results of the dual EKF estimator are promising. As shown in Fig. 7, the estimation results of the dual EKF method vary within $6.8 \%$ for all the applied test cases as illustrated in Table 2 With this performance, the dual EKF is superior to the standard EKF approach, presented in [17], for most of the cases with precise initial conditions for the SoC state. However, the most significant improvement of the dual EKF lays in its behaviour without precise initial conditions. While the standard EKF takes the whole discharge process to converge to the reference $\mathrm{SoC}$ when the starting point of the estimator is set to $S_{o} C_{0}=0.6$, the dual EKF converges in all cases within seconds. This is the reason why the dual EKF for $S o C_{0}=0.6$ is not drawn as a separate line in Fig. 7. For the presented time scale, both graphs of the dual EKF, for the precise and imprecise initial conditions, are similar. Here, the assignment of different covariance values to the plateaus improved the convergence significantly, which is possible due to the simple distinction of the two voltage plateaus of Li-S cells. The relative constant values for the estimation error over the whole discharge range for all tests and initial conditions is a sign for the robustness of the estimation.

The largest estimation errors occur for the higher current density cases. Here the SoC estimation slowly drifts to $9.5 \%$ error towards the end of discharge 

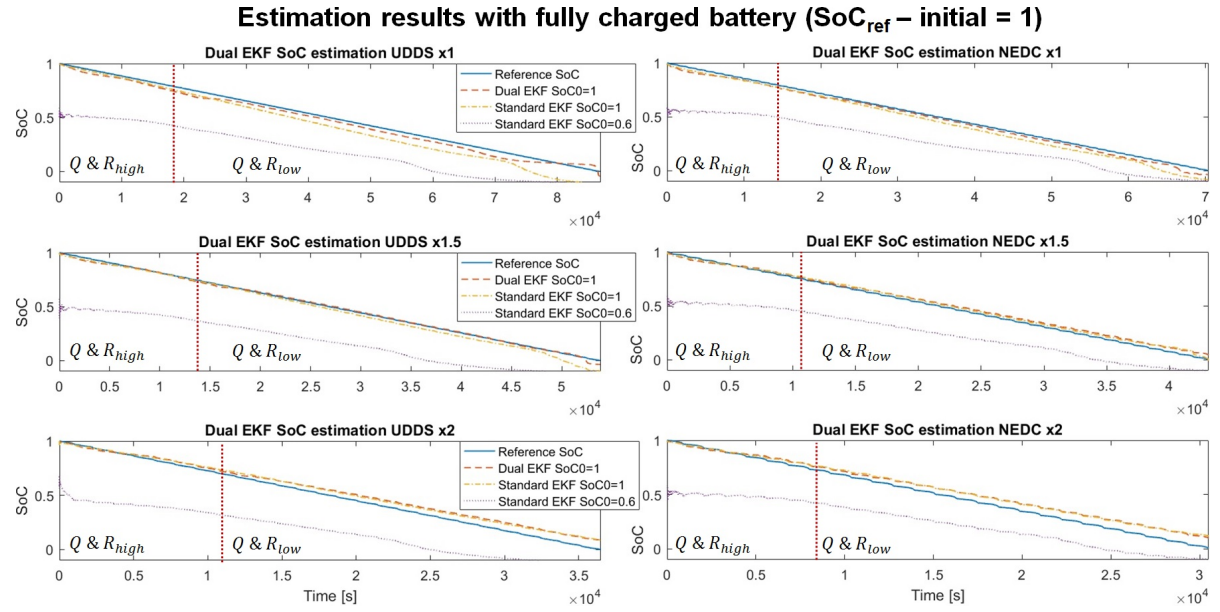

Figure 7: Results of the SoC estimation with different current profiles and rates

(NEDC $x 2$ ). This is caused by the emphasis on the Coulomb counting within the low plateau and could be improved by better utilisation of the internal resistance growth. However, with an average error of about $6.8 \%$ in the worst case of all six SoC estimation evaluation tests, the approach is considered as sufficiently robust (see Table 2). 
Table 2: Root-Mean-Square Error (RMSE) in SoC estimation with UDDS and NEDC current profile with different current scaling gains for an initially fully-charged battery. The estimator was initialized with SoC values of $1,0.7$ and 0.6 .

\begin{tabular}{c|c|ccc|ccc}
\hline \multirow{2}{*}{$\begin{array}{c}\text { current } \\
\text { scaling }\end{array}$} & $\begin{array}{c}\text { initial } \\
\text { SoC } \\
\text { estimate }\end{array}$ & $\begin{array}{c}\text { dual EKF } \\
\text { (new) }\end{array}$ & $\begin{array}{c}\text { single EKF } \\
\text { (old) }\end{array}$ & $\begin{array}{c}\text { improve- } \\
\text { ment }\end{array}$ & $\begin{array}{c}\text { NEDC driving cycle } \\
\text { dual EKF } \\
\text { (new) }\end{array}$ & $\begin{array}{c}\text { single EKF } \\
\text { (old) }\end{array}$ & $\begin{array}{c}\text { improve- } \\
\text { ment }\end{array}$ \\
\hline \multirow{3}{*}{$\times 1$} & 1 & 0.0346 & 0.0827 & $58.2 \%$ & 0.0257 & 0.0490 & $47.6 \%$ \\
& 0.7 & 0.0346 & 0.0832 & $58.4 \%$ & 0.0257 & 0.0519 & $50.5 \%$ \\
& 0.6 & 0.0455 & 0.3215 & $85.8 \%$ & 0.0350 & 0.2600 & $86.5 \%$ \\
\hline \multirow{3}{*}{$\times 1.5$} & 1 & 0.0118 & 0.0334 & $64.7 \%$ & 0.0274 & 0.0217 & $-26.3 \%$ \\
& 0.7 & 0.0118 & 0.0360 & $67.2 \%$ & 0.0274 & 0.0263 & $-4.2 \%$ \\
& 0.6 & 0.0360 & 0.3370 & $89.3 \%$ & 0.0336 & 0.2721 & $87.7 \%$ \\
\hline \multirow{3}{*}{$\times 2$} & 1 & 0.0523 & 0.0478 & $-9.4 \%$ & 0.0581 & 0.0580 & $-0.2 \%$ \\
& 0.7 & 0.0523 & 0.0495 & $-5.7 \%$ & 0.0581 & 0.0606 & $4.1 \%$ \\
& 0.6 & 0.0556 & 0.3463 & $83.9 \%$ & 0.0680 & 0.2814 & $75.8 \%$ \\
\hline
\end{tabular}




\subsection{SoC estimation with an initially partly discharged battery}

Improvements are also visible for the estimation with partly discharged battery. Here, the simulation starts at 0.6 reference SoC, which is roughly $10 \%$ lower than the transition point in between both voltage plateaus. This scenario is more realistic since the Li-S battery is likely to self discharge when the SoC monitoring system is switched off. For this simulation the initial conditions of the online parametrization EKF are not changed (Eq. 26). For this scenario however, the values are highly imprecise. Furthermore, the initial SoC of the second EKF is set to 1 .

The first output of the test is that the online parametrization is robust against imprecise initial conditions. The $\mathrm{OCV}$ and internal resistance converge in all six cases to steady values within $50 \mathrm{~s}$ to $100 \mathrm{~s}$ simulation time, which is particular useful for the SoC estimation since it relies on precise parameters. And indeed, the results presented in Fig. 8 and Table 3 show that the SoC also converges within the same period. However, the convergence towards the reference SoC stops at the transition point of the voltage plateaus, roughly $10 \%$ over the reference SoC.
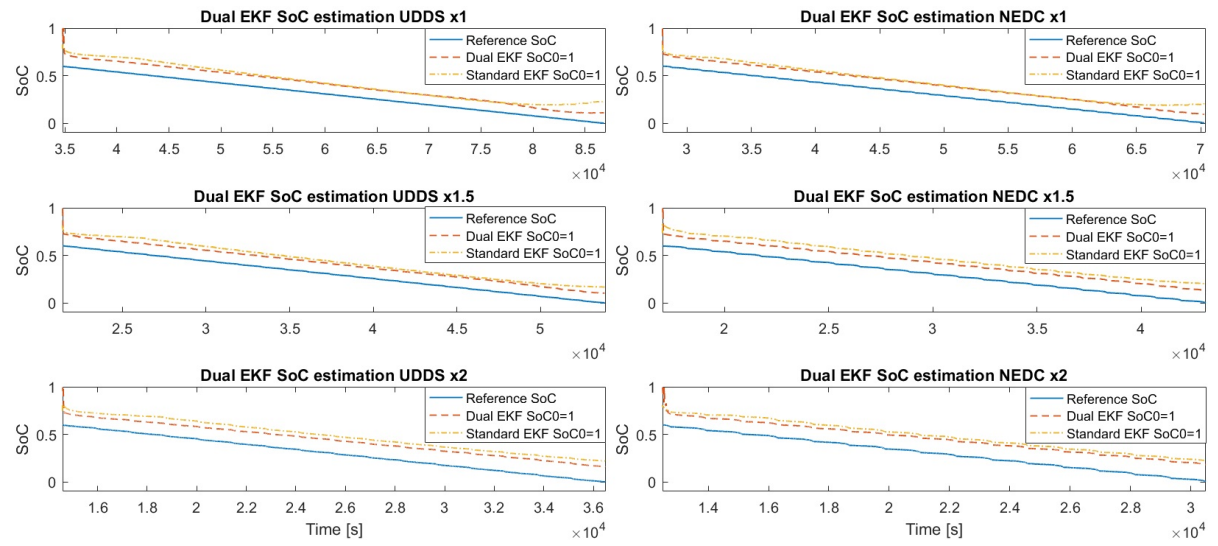

Figure 8: Results of the SoC estimation with different current profiles and rate densities with partly discharged battery

After the identified OCV falls under $2.15 \mathrm{~V}$ the estimation changes its emphasis on Coulomb counting. This means that the correction of the state 
Table 3: Root-Mean-Squared Error (RMSE) in SoC estimation with UDDS and NEDC current profile with different current scaling gains for an initially partly-discharged battery. In all cases, the estimator was initialized with a SoC estimate of 1 , though the true initial SoC was 0.6.

\begin{tabular}{|c|c|c|c|c|c|c|}
\hline \multirow[b]{2}{*}{$\begin{array}{l}\text { current } \\
\text { scaling }\end{array}$} & \multicolumn{3}{|c|}{ UDDS driving cycle } & \multicolumn{3}{|c|}{ NEDC driving cycle } \\
\hline & $\begin{array}{l}\text { dual EKF } \\
\text { (new) }\end{array}$ & $\begin{array}{l}\text { single EKF } \\
\text { (old) }\end{array}$ & $\begin{array}{l}\text { improve- } \\
\text { ment }\end{array}$ & $\begin{array}{c}\text { dual EKF } \\
\text { (new) }\end{array}$ & $\begin{array}{l}\text { single EKF } \\
\text { (old) }\end{array}$ & $\begin{array}{l}\text { improve- } \\
\text { ment }\end{array}$ \\
\hline$\times 1$ & 0.1061 & 0.1323 & $19.8 \%$ & 0.1030 & 0.1232 & $16.4 \%$ \\
\hline$\times 1.5$ & 0.1100 & 0.1421 & $22.6 \%$ & 0.1214 & 0.1691 & $28.2 \%$ \\
\hline$\times 2$ & 0.1422 & 0.1890 & $24.8 \%$ & 0.1538 & 0.1860 & $17.3 \%$ \\
\hline
\end{tabular}

is slowly and can only be seen for the lower currents (UDDS $\times 1, \mathrm{NEDC} \times 1$ ). Since the starting point of the simulation was chosen about $10 \%$ SoC beneath the transition point, also the estimation errors for this case are in that region. The slow convergence of the SoC estimation within the low plateau is one disadvantage of the presented method. However, the properties of Li-S cells help to keep the estimation error within reasonable rages. The self discharge is expected to be present only in the high plateau [14], which founds the assumption that a self discharged Li-S battery is likely to be near the transition point between both plateaus for a long time. Therefore, the presented limitation is expected to be small for most applications.

\subsection{SoC estimation with multiple cycles}

So far we presented the improved estimation accuracy and robustness against initial conditions for the dual EKF approach. To emphasize the advantages, we also present one of our experiments with three subsequent NEDC cycles with a constant charge of $0.32 \mathrm{~A}$ in between. Here, we demonstrate the usefulness of the dual EKF estimation, despite the lack of a charging model or a deep understanding of the charging process.

As simple additions necessary for the estimator to cope with the charging an assumed linear decay of the internal resistance, a coulombic efficiency of 
0.9 and heavily emphasized Coulomb counting during charging are added

$$
R_{\text {charge }}=\left[\begin{array}{cc}
0.00015 & 0 \\
0 & 55
\end{array}\right], Q_{\text {charge }}=\left[\begin{array}{cc}
0.1 & 0 \\
0 & 0.0000000001
\end{array}\right]
$$

The slow descent of the $R_{\text {int }}$ is assumed because the constant charge current does not allow the parameter estimation to adjust automatically. Tests in Fig. 9 revealed a variation in the discharge capacity, variances in the charging efficiency and an unknown (not modelled) charge curve. Here, it is visible how powerful the concept of uncertainty is for the state estimation. During charge the SoC estimator can roughly estimate the SoC through Coulomb counting, while the uncertainties adjust for inaccuracies of the model or differences in the battery behaviour. Especially in the high plateau the correction works well and the drift from the crude charging assumptions is corrected within seconds after the discharge starts again. After the second charge the single EKF method has roughly the same error than the dual EKF, but needs considerable more time to converge back to the reference $\mathrm{SoC}$ in the subsequent discharge. The overall accuracy (RMSE) of the dual EKF for the entire test period is 0.0450 and can be seen as accurate enough for most applications. However, it has to be invested more effort to understand the charging process to ensure the results are robust for more diverse user cases. Furthermore, the robustness has to be proven with drive cycles that assume a recuperation of kinetic energy during the drive cycle. 

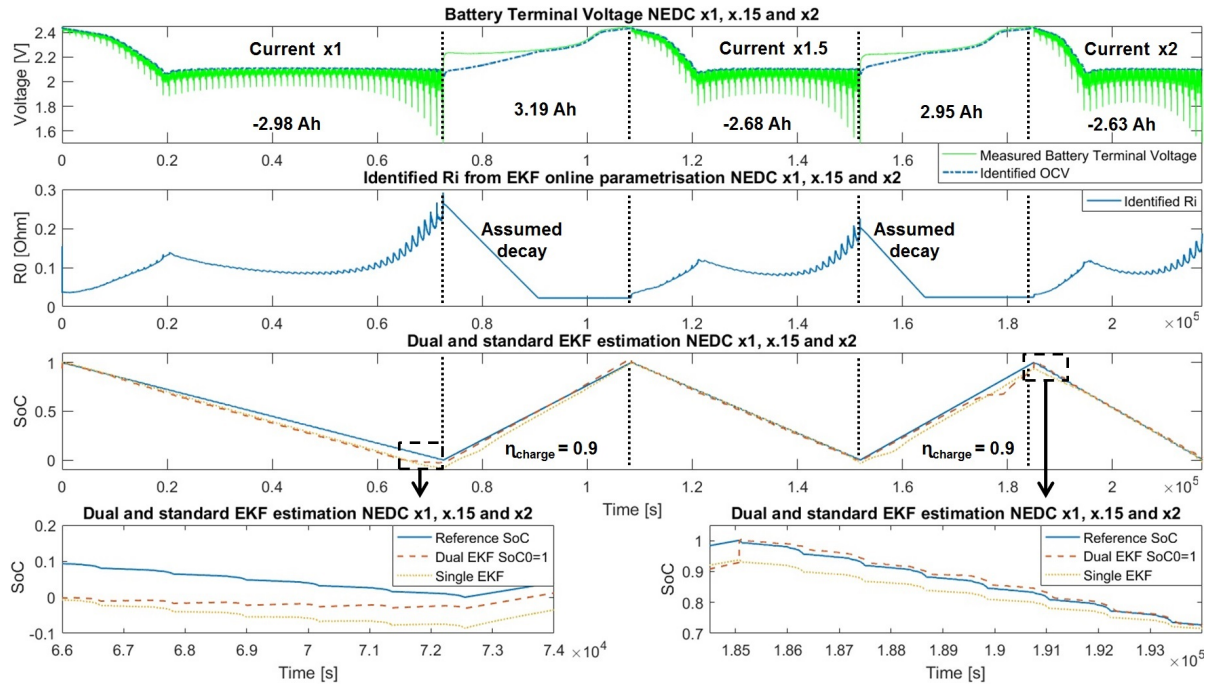

Figure 9: Results of the SoC estimation with three NEDC current profiles with constant charge

\section{Conclusion}

This study has explored the potential for a previously unapplied architecture for SoC estimation in Li-S batteries using a 'dual extended Kalman filter' architecture, with a first-stage filter estimating model parameters online and a second-stage filter using these parameter estimates to form a SoC estimate. Key findings are as follows:

- It has been shown shown that an online parameter estimation with an extended Kalman filter can identify the parameters of an behavioural interpretation of a Thevenin equivalent circuit reliably. This method is fast enough to operate in real-time, and can be used to identify model parameters in continuous operation. The current profiles must change dynamically for this method to work, but this will be the case with many practical current profiles changes. The method has been demonstrated with two automotive driving cycles, the NEDC and the UDDS.

- The new parameter estimation method has been applied to scaled driving cycle current profiles: three different scaling factors were used for each 
of the NEDC and the UDDS. This showed the relationship between discharge current rate and the real-time estimate of internal resistance. The relationship discovered was consistent with that expected from the electrochemistry, as described in the literature. To model these effects in a practical BMS application and improve estimator accuracy with diverse currents, a new model was developed incorporating a dynamic internal resistance term.

- The new estimator was used within a 'dual extended Kalman filter' architecture to give robust SoC estimation: the online estimates of $\mathrm{OCV}$ and internal resistance from the first extended Kalman filter were used as inputs to a second Kalman filter, modelling the relationship between equivalent circuit parameters and state of charge. The results were compared to those obtained in the literature for a single-stage estimator, and it was found that the new method offered greater accuracy in almost all cases. Compared to the previous methods, the new methods were particularly beneficial when there were large deviations in the current scaling from the nominal level. The accuracy and robustness of the estimation results demonstrate the effectiveness of employing battery model parameters for SoC estimation.

There are a number of potential future directions that could be taken. One would be to use better models with a stronger electrochemical basis. To date, only equivalent circuit network models have been used for state estimation, partly because they are computationally tractable, but also because there are few fast-operating electrochemical models for Li-S. This situation is changing, and it would be beneficial to explore the potential of new fast 'low dimensional' electrochemical models for state of charge estimation. This would potentially improve state estimation further, and it could also enhance understanding of the 'in application' reaction path. Related to this, it would be beneficial to extend state estimation from state of charge to state of health measures, giving an indication of the degradation of capacity and power in operation. This could 
be done either through fast electrochemical models or through appropriate adaptions to the behavioural model.

\section{Acknowledgement}

This research was undertaken as part of the project 'Revolutionary Electric Vehicle Battery' (REVB), co-funded by Innovate UK under grant TS/L000903/1; university funding is provided by EPSRC under grant number EP/L505286/1. The data used in this article is described at $10.17862 /$ cranfield.rd.c.3723934 it is subject to an embargo, and will be available from May 2022.)

\section{References}

[1] V. Kolosnitsyn, E. Karaseva, Lithium-sulfur batteries: Problems and solutions, Russian Journal of Electrochemistry 44 (2008) 506-509.

[2] Y. Diao, K. Xie, S. Xiong, X. Hong, Shuttle phenomenon-the irreversible oxidation mechanism of sulfur active material in Li-S battery, Journal of Power Sources 235 (2013) 181-186.

[3] X. Ji, L. F. Nazar, Advances in Li-S batteries, Journal of Materials Chemistry 20 (2010) 9821-9826.

[4] H. Yamin, E. Peled, Electrochemistry of a nonaqueous lithium/sulfur cell, Journal of Power Sources 9 (1983) 281-287.

[5] Y. V. Mikhaylik, J. R. Akridge, Polysulfide shuttle study in the Li/S battery system, Journal of the Electrochemical Society 151 (2004) A1969-A1976.

[6] M. U. Patel, R. Demir-Cakan, M. Morcrette, J.-M. Tarascon, M. Gaberscek, R. Dominko, Li-s battery analyzed by UV/Vis in Operando mode, ChemSusChem 6 (2013) 1177-1181. 
[7] Y. V. Mikhaylik, I. Kovalev, R. Schock, K. Kumaresan, J. Xu, J. Affinito, High energy rechargeable Li-S cells for EV application: status, remaining problems and solutions, Ecs Transactions 25 (2010) 23-34.

[8] M. Marinescu, T. Zhang, G. J. Offer, A zero dimensional model of lithiumsulfur batteries during charge and discharge, Physical Chemistry Chemical Physics 18 (2016) 584-593.

[9] M. Wild, L. O'Neill, T. Zhang, R. Purkayastha, G. Minton, M. Marinescu, G. J. Offer, Lithium sulfur batteries, a mechanistic review, Energy \& Environmental Science 8 (2015) 3477-3494.

[10] H. S. Ryu, Z. Guo, H. J. Ahn, G. B. Cho, H. Liu, Investigation of discharge reaction mechanism of lithium | liquid electrolyte I sulfur battery, Journal of Power Sources 189 (2009) 1179-1183.

[11] M. R. Busche, P. Adelhelm, H. Sommer, H. Schneider, K. Leitner, J. Janek, Systematical electrochemical study on the parasitic shuttle-effect in lithium-sulfur-cells at different temperatures and different rates, Journal of Power Sources 259 (2014) 289-299.

[12] V. Kolosnitsyn, E. Kuzmina, E. Karaseva, On the reasons for low sulphur utilization in the lithium-sulphur batteries, Journal of Power Sources 274 (2015) 203-210.

[13] V. Knap, D. I. Stroe, R. Purkayastha, S. Walus, D. J. Auger, A. Fotouhi, K. Propp, Methodology for assessing the lithium-sulfur battery degradation for practical applications, ECS Transactions 77 (2017) 479-490.

[14] V. Knap, D.-I. Stroe, M. Swierczynski, R. Teodorescu, E. Schaltz, Investigation of the self-discharge behavior of lithium-sulfur batteries, Journal of The Electrochemical Society 163 (2016) A911-A916.

[15] C. E. Parfitt, Characterisation, modelling and management of lithiumsulphur batteries for spacecraft applications (2012). 
[16] K. Propp, M. Marinescu, D. J. Auger, L. O’Neill, A. Fotouhi, K. Somasundaram, G. J. Offer, G. Minton, S. Longo, M. Wild, V. Knap, Multi-temperature state-dependent equivalent circuit discharge model for lithium-sulfur batteries, Journal of Power Sources 328 (2016) 289-299.

[17] K. Propp, D. J. Auger, A. Fotouhi, S. Longo, V. Knap, Kalman-variant estimators for state of charge in lithium-sulfur batteries, Journal of Power Sources 343 (2017) 254-267.

[18] G. L. Plett, Extended Kalman filtering for battery management systems of LiPB-based HEV battery packs: Part 1. background, Journal of Power sources 134 (2004) 252-261.

[19] Y.-H. Chiang, W.-Y. Sean, J.-C. Ke, Online estimation of internal resistance and open-circuit voltage of lithium-ion batteries in electric vehicles, Journal of Power Sources 196 (2011) 3921-3932.

[20] L. Thévenin, Extension de la loi d'Ohm aux circuits électromoteurs complexes [extension of Ohm's law to complex electromotive circuits], in: Annales Télégraphiques, volume 10, 1883, pp. 222-224.

[21] H. He, R. Xiong, H. Guo, S. Li, Comparison study on the battery models used for the energy management of batteries in electric vehicles, Energy Conversion and Management 64 (2012) 113-121.

[22] A. Fotouhi, D. J. Auger, K. Propp, S. Longo, Accuracy versus simplicity in online battery model identification, IEEE Transactions on Systems, Man, and Cybernetics: Systems (2016).

[23] H. He, R. Xiong, H. Guo, Online estimation of model parameters and state-of-charge of LiFePO 4 batteries in electric vehicles, Applied Energy 89 (2012) 413-420.

[24] R. Xiong, H. He, F. Sun, K. Zhao, Evaluation on state of charge estimation of batteries with adaptive extended Kalman filter by experiment approach, IEEE Transactions on Vehicular Technology 62 (2013) 108-117. 
[25] A. Rosenman, R. Elazari, G. Salitra, E. Markevich, D. Aurbach, A. Garsuch, The effect of interactions and reduction products of lino3, the anti-shuttle agent, in Li-S battery systems, Journal of The Electrochemical Society 162 (2015) A470-A473.

[26] A. Ferrese, J. Newman, Modeling lithium movement over multiple cycles in a lithium-metal battery, Journal of The Electrochemical Society 161 (2014) A948-A954.

[27] D. Simon, Kalman filtering, Embedded systems programming 14 (2001) 72-79.

[28] G. Welch, G. Bishop, An introduction to the Kalman filter. Department of Computer Science, University of North Carolina, 2006.

[29] G. L. Plett, Extended Kalman filtering for battery management systems of LiPB-based HEV battery packs, Journal of Power Sources 134 (2004) 252-261.

[30] G. L. Plett, Sigma-point Kalman filtering for battery management systems of LiPB-based HEV battery packs, Journal of Power Sources 161 (2006) 1356-1368.

[31] S. Samuel, L. Austin, D. Morrey, Automotive test drive cycles for emission measurement and real-world emission levels - a review, Proceedings of the Institution of Mechanical Engineers, Part D: Journal of Automobile Engineering 216 (2002) 555-564.

[32] R. Kötz, S. Müller, M. Bärtschi, B. Schnyder, P. Dietrich, F. Büchi, A. Tsukada, G. Scherer, P. Rodatz, O. Garcia, et al., Supercapacitors for peak-power demand in fuel-cell-driven cars, in: ECS Electro-Chemical Society, 52nd Meeting,, San Francisco, 2001, pp. 564-575.

[33] L. Ljung, System identification: Theory for the user, PTR Prentice Hall Information and System Sciences Series 198 (1987). 
[34] M. Fellah, G. Singh, A. Rousseau, S. Pagerit, E. Nam, G. Hoffman, Impact of real-world drive cycles on PHEV battery requirements, Technical Report, SAE Technical Paper, 2009.

[35] A. Jossen, Fundamentals of battery dynamics, Journal of Power Sources 154 (2006) 530-538.

[36] V. Kolosnitsyn, E. Kuzmina, E. Karaseva, S. Mochalov, A study of the electrochemical processes in lithium-sulphur cells by impedance spectroscopy, Journal of Power Sources 196 (2011) 1478-1482.

[37] Z. Deng, Z. Zhang, Y. Lai, J. Liu, J. Li, Y. Liu, Electrochemical impedance spectroscopy study of a lithium/sulfur battery modeling and analysis of capacity fading, Journal of The Electrochemical Society 160 (2013) A553-A558.

[38] A. Fotouhi, D. J. Auger, K. Propp, S. Longo, Electric vehicle battery parameter identification and SOC observability analysis: NiMH and Li-S case studies, IET Power Electronics 10 (2017) 1289-1297.

[39] V. Knap, D.-I. Stroe, R. Teodorescu, M. Swierczynski, T. Stanciu, Electrical circuit models for performance modeling of lithium-sulfur batteries, in: Energy Conversion Congress and Exposition (ECCE), 2015 IEEE, IEEE, 2015, pp. 1375-1381.

[40] J. Xu, C. C. Mi, B. Cao, J. Deng, Z. Chen, S. Li, The state of charge estimation of lithium-ion batteries based on a proportional-integral observer, Vehicular Technology, IEEE Transactions on 63 (2014) 1614-1621.

[41] A. Fotouhi, D. J. Auger, K. Propp, S. Longo, M. Wild, A review on electric vehicle battery modelling: From lithium-ion toward lithium-sulphur, Renewable and Sustainable Energy Reviews 56 (2016) 1008-1021.

[42] MathWorks, MATLAB version 8.5.0.197613 (R2015a), 2015. 
[43] T. Zhang, M. Marinescu, L. O'Neill, M. Wild, G. Offer, Modeling the voltage loss mechanisms in lithium-sulfur cells: the importance of electrolyte resistance and precipitation kinetics, Physical Chemistry Chemical Physics 17 (2015) 22581-22586. 
2019-10-23

\title{
Improved state of charge estimation for lithium-sulfur batteries
}

\author{
Propp, Karsten
}

Elsevier

Propp K, Auger DJ, Fotouhi A, et al., (2019) Improved state of charge estimation for

lithium-sulfur batteries. Journal of Energy Storage, Volume 26, December 2019, Article number 100943

https://doi.org/10.1016/j.est.2019.100943

Downloaded from Cranfield Library Services E-Repository 